\title{
Le programme international "Faisabilité de l'évacuation des déchets de haute activité sous les fonds marins" (programme "Seabed")
}

\author{
A. BARBREAU*
}

(Manuscrit reçu le 7 février 1990)

RÉSUMÉ Le programme faisabilité "Seabed" est un programme scientifique international de recherche sur la faisabilité de l'évacuation des déchets radioactifs de haute activité dans les formations géologiques constituant le fond des grandes plaines abyssales des océans. Décidé en 1977, ce programme se proposait de répondre aux trois questions sulvantes : Existe-t-il des sites potentiellement favorables ? La mise en place des déchets est-elle possible ? L'opération présente-t-elle toutes les garanties de sûreté ? Initié au départ par quatre pays, les Etats-Unis, le Royaume-Uni, le Japon et la France, ce programme, patronné par I'AEN de l'OCDE, rassemblait en 1988 dix pays et la Commission des Communautés européennes.

Les techniques de mise en place des déchets au moyen de forages dans les sédiments consolidés et au moyen de torpilles (pénétreurs) dans les sédiments meubles ont été examinées. La technique des torpilles a fait l'objet des études les plus poussées et notamment d'expériences in situ dans l'océan Atlantique. Les différents facteurs intervenant dans la sûreté ont été étudiés et les conséquences radiologiques d'une opération d'enfouissement évaluées au moyen de modèles. La conclusion est qu'une telle opération pourrait être réalisée techniquement dans des conditions de sâreté tout à fait satisfaisantes.

ABSTRACT The "Seabed" feasibility program is an international scientific program of research on the feasibility of the disposal of high-level radioactive wastes into the geological formations making up the floor of the great abyssal plains of the oceans. Decided in 1977, the program is aimed at answering the three following questions : 1) are there potentially favourable sites ? 2) is the disposal of wastes possible ? 3) does the operation present safety guarantees ?

First initiated by four countries (USA, UK, Japan and France), the program sponsored by the OECD nuclear energy agency was gathering ten countries and the Commission of the European communities in 1988. The techniques of waste disposal by means of drilling in consolidated sediments and penetrators in loose sediments have been studied. The penetrator technique has been the most thoroughly studied, especially through in situ experiments in the Atlantic ocean. The various factors affecting safety have been studied and the radiological consequences of a burial operation assessed through models. It has been concluded that such an operation could be carried out technically under quite satisfying conditions.

\footnotetext{
* Commissariat à l'énergie atomique, Institut de protection et de sûreté nucléaire, Département de protection technique, BP 6, 92265 Fontenay-aux-Roses Cedex.

A. BARBREAU a été membre du comité exécutif du Groupe international Seabed depuis sa fondation en 1977 et le coordonnateur du programme français durant son déroulement.
} 


\section{INTRODUCTION}

Le stockage des déchets radioactifs sous forme solide, sous le fond des océans, dans les sédiments ou autres formations géologiques sousmarines repose sur des concepts de base identiques à ceux sur lesquels s'appuie l'évacuation en formation géologique continentale, la solution actuellement retenue et en cours d'études actives dans un certain nombre de pays :

- isolement et confinement des déchets dans des structures imperméables ou très peu perméables, stables durant de longues périodes de temps,

- systèmes multi-barrière(s), dans lesquels la barrière géologique joue le rôle essentiel pour la sûreté,

- passivité du système qui, abandonné à lui-même, ne nécessite ni contrôle ni surveillance.

Mais l'option "formations géologiques sous-marines" présente, en plus, des avantages importants qui lui sont propres :

- les fonds océaniques constituent des structures relativement stables : leur formation est en relation avec la tectonique des plaques et résulte de l'extrusion de basalte le long des rifts (dans l'océan Atlantique, le long de la dorsale atlantique) et ils sont, par conséquent, d'autant plus anciens qu'ils sont plus éloignés du rift ; durant des millions d'années, des sédiments se sont déposés sur cette croûte basaltique, en dehors des grands mouvements orogéniques qui ont affecté le milieu continental, soit par apport de sédiments depuis la surface ou depuis les marges continentales, soit à la faveur de courant de turbidité, les plus anciens dépôts sont maintenant consolidés et sont souvent recouverts de sédiments meubles ou faiblement consolidés qui peuvent être épais de plusieurs centaines de mètres dans certaines plaines abyssčles ;

- ces formations sédimentaires sont étendues et isolées sous plusieurs kilomètres d'eau ;

- on peut supposer une absence de gradient de charge hydraulique, puisque l'eau interstitielle contenue dans les sédiments doit être en équilibre hydrostatique avec la masse d'eau océanique ; les seuls mécanismes possibles pour mettre en mouvement les eaux interstitielles pourraient être des gradients thermiques ou l'expulsion d'eau par compaction de sédiments ;

- enfin, l'énorme volume d'eau que représente la masse océanique constitue, par ses capacités de dilution, un volant additionnel de sécurité dans le cas où la barrière sédimentaire laisserait échapper quelques fuites.

II était donc naturel que l'on s'intéressât à cette option a priori attrayante, notamment du point de vue de la sûreté, et qui peut donc constituer une alternative à l'évacuation des déchets radioactifs dans les formations géologiques continentales. 
Rapidement, il est apparu que deux options techniques se présentaient pour la mise en place des déchets radioactifs à l'intérieur des sédiments en fonction de la consistance de ces derniers :

- dans le cas de sédiments meubles, la technique la plus simple et la plus économique consiste à enfermer les déchets dans des torpilles qui, larguées depuis la surface de l'océan, s'enfonceraient, grâce à la vitesse acquise, jusqu'à des profondeurs de plusieurs dizaines de mètres :

- dans le cas des sédiments consolidés ou de la croûte basaltique, on ne peut envisager que de faire appel à la technique des forages, en adaptant, par exemple, les méthodes de foration auxquelles on a recours en océanographie avec des navires comme le GLOMAR CHALLENGER dans le passé et maintenant son successeur, le navire américain "RESOLUTION".

\section{HISTORIQUE}

Le milieu océanique étant en très grande partie international, le sujet se prêtait particulièrement à une coopération entre différents pays. La possibilité d'engager une coopération internationale dans le domaine de l'évacuation des déchets radioactifs de haute activité dans les fonds marins a été discutée, pour la première fois, lors de la première session du Comité de gestion des déchets radioactifs de l'Agence pour l'énergie nucléaire (OCDE) en octobre 1975. Les Etats-Unis qui avaient en juin 1973 lancé un programme dans ce domaine (Laboratoires SANDIA), étaient particulièrement intéressés à voir d'autres pays s'y associer, tant pour des raisons techniques et financières que politiques ; ils ont donc organisé une réunion internationale à l'Institut océanographique de Woods-Hole, en février 1976, pour discuter de la question et d'une éventuelle coopération. Elle a rassemblé des participants des pays suivants : Etats-Unis, France, Royaume-Uni, Australie, Canada, République fédérale d'Allemagne, Commission des Communautés européennes (CCE).

La réunion se proposait de répondre aux quatre questions suivantes :

- Le projet a-t-il un intérêt ?

- Y a-t-il, au stade actuel, des contre-indications ?

- Quelles pourraient en être les grandes lignes ?

- Qui est intéressé à participer ?

A la suite de cette réunion où, durant une semaine, fut discuté l'état des connaissances en la matière, il fut conclu que le projet était très intéressant et qu'il n'y avait pas de contre-indication, à la lumière des connaissances scientifiques actuelles. Toutefois, la faisabilité de l'option Seabed restait à démontrer : en effet, les fonds océaniques étaient encore, à ce moment là, mal connus. 
En dehors de dragages très superficiels, seul, un nombre limité de sondages, réalisés dans le cadre de grands programmes internationaux de recherche océanographique tels que le projet IPOD, avait permis de reconnaître les sédiments des grands fonds océanographiques, dont le mode de formation et la nature réelle n'ont commencé à être vraiment connus que depuis quelques décennies, à la lumière de la tectonique des plaques.

Il était donc nécessaire de développer un important programme de recherches portant sur la possibilité de mettre en place les conteneurs radioactifs dans les sédiments sous une grande épaisseur d'eau, de trouver des sites favorables, garantissant la sûreté de l'opération, et de développer les modèles nécessaires à l'évaluation de sûreté.

Finalement, quatre pays ont, immédiatement après la réunion de Woods-Hole, fait part de leur intention de participer à une action commune : Les Etats-Unis, le Royaume-Uni, le Japon et la France.

En mars 1977, une réunion à laquelle participèrent ces quatre pays et un représentant de l'AEN, se tint à Washington, à l'invitation des USA, au cours de laquelle furent posées les bases de la collaboration internationale projetée : création d'un groupe de travail (working group SEABED "WGS") comprenant un comité exécutif et plusieurs sous-groupes ou groupes techniques d'actions (task groups) ayant en charge différents domaines d'études : la colonne d'eau, le conteneur, la forme des déchets, la biologie, l'analyse des systèmes et modélisation, les sédiments et les roches et les critères de choix de sites. Chaque pays a désigné un représentant au comité exécutif et dans chacun des groupes techniques, chaque groupe technique ayant un animateur coopté parmi ses membres et ayant pour mission de développer dans son domaine propre les échanges d'informations et la collaboration.

Les programmes, sous la responsabilité des états membres, devaient viser à répondre aux trois questions fondamentales suivantes :

- existe-t-il des sites favorables?

- est-il techniquement possible de mettre en place les conteneurs à la profondeur appropriée et quelles sont les conséquences de cette mise en place sur la capacité de confinement de la barrière sédimentaire ?

- quelles sont les conséquences radiologiques d'une opération d'évacuation de déchets de haute activité dans les sédiments sous-marins profonds ?

Le groupe de travail "SEABED" s'est défini les principaux objectifs suivants :

- organiser les contacts pour des discussions, évaluer les progrès accomplis et organiser les efforts futurs ;

- encourager et coordonner des campagnes à la mer et des expériences entre les nations participantes, avec la possibilité d'organiser des campagnes d'études communes dans l'avenir ; 
- partager les moyens d'études et les équipements ;

- échanger des données dans le domaine de l'océanographie et de l'ingéniérie ;

- se tenir au courant des questions de politique internationale en la matière.

Il a été admis que les résultats des recherches effectuées dans le cadre de ce programme seraient mis à la disposition de tous les pays participants et que chacun s'efforcerait d'harmoniser son propre programme avec celui des autres, de telle sorte que les différents programmes couvrent l'ensemble des besoins scientifiques pour atteindre l'objectif visé dans les meilleures conditions économiques.

Le WGS s'est constitué comme un groupe restreint de l'AEN, composé de pays ayant un programme actif et d'accord entre eux pour coordonner leur programme. Tout pays répondant à ces conditions pouvait y adhérer : il lui suffisait d'en informer le secrétariat de l'AEN par l'intermédiaire de son représentant au comité de gestion de l'AEN. Les programmes techniques devaient relever exclusivement des pays participants, entre qui les échanges d'informations seraient limités. Le WGS informerait le comité de gestion des déchets radioactifs de l'AEN de ses activités, au moyen d'un rapport annuel.

Les réunions suivantes se tînrent : en 1978 et 1979 à Albuquerque (USA), en 1980 à Bristol (Royaume-Uni), en 1981 à Paris (France), en 1982 à SanDiego-la-Jolla (USA), en 1983 à Varese (Italie), en 1984 à Berlin (RFA), en 1985 à Halifax (Canada), en 1986 à Urbino (Italie), en 1987 à Tokyo et Mito (Japon).

La réunion au Japon a été essentiellement consacrée au rapport de faisabilité dont les premières moutures, préparées depuis la réunion d'Urbino, ont été activement discutées.

Au cours des années, le nombre de pays participants s'est accru tant comme participants actifs que comme associés ; à la fin, le groupe comprenait : la République fédérale d'Allemagne, la Belgique, le Canada, la CCE, les USA, la France, l'Italie, le Japon, les Pays-Bas, le Royaume-Uni et la Suisse.

Au début des années 1980, le groupe a été restructuré. Le besoin s'est, en effet, alors, fait sentir d'assurer une meilleure coordination des études menées dans les différents pays en vue d'aboutir, dans un délai raisonnable, à une évaluation de la faisabilité du concept. Au comité exécutif et aux groupes techniques (eux-mêmes restructurés) s'est ajouté un échelon intermédiaire : le bureau de coordination. L'organisation s'est alors présentée de la façon suivante:

COMITÉ DE GESTION DES DÉCHETS RADIOACTIFS Comité exécutif du groupe de travall sur l'évacuation sous les fonds marins Comite executif du greau de coordination
Bupe de travall sur l'evacu

\begin{tabular}{|c|c|c|c|c|c|c|}
\hline $\begin{array}{c}\text { Sous-groupe } \\
\text { sur } \\
\text { l'évaluation } \\
\text { des sites }\end{array}$ & $\begin{array}{c}\text { I } \\
\text { Sous-groupe } \\
\text { sur } \\
\text { les études } \\
\text { techniques }\end{array}$ & $\begin{array}{l}\text { Sous-groupe } \\
\text { sur } \\
\text { le champ } \\
\text { proche }\end{array}$ & $\begin{array}{c}\text { Sous-groupe } \\
\text { sur } \\
\text { la barrière } \\
\text { sédimentaire }\end{array}$ & $\begin{array}{c}\text { Sous-groupe } \\
\text { sur } \\
\text { Pocéanographie } \\
\text { physique }\end{array}$ & $\begin{array}{c}\text { Sous-groupe } \\
\text { sur } \\
\text { l'océanographie } \\
\text { biologique }\end{array}$ & $\begin{array}{c}\text { Sous-groupe } \\
\text { sur } \\
\text { p'evaluation } \\
\text { biologique }\end{array}$ \\
\hline
\end{tabular}


En 1982, le groupe a lancé un plan quinquennal qui devait s'étendre sur la période 1983-1987, en vue de rassembler le maximum d'informations dans les différents domaines techniques recouverts par les sousgroupes et pour qu'il soit aussi possible, en fin de plan, de faire la synthèse des résultats dans un rapport consacré à la faisabilité de l'évacuation des déchets radioactifs dans les fonds marins, rapport destiné au comité de gestion de l'AEN. Les objectifs spécifiques de ce plan quinquennal étaient les suivants :

- sélectionner un site de référence pour les études de faisabilité et d'impact ;

- évaluer la faisabilité technique et les coûts sur le site de référence ;

- calculer l'impact radiologique.

On a pu exploiter les résultats des études réalisées auparavant et développer de nouvelles recherches dans les domaines à préciser ou à élucider.

Les USA et le Royaume-Uní ont été les pays qui ont le plus investi dans le programme SEABED depuis le début, sur le plan financier ; il s'agissait pour eux et notamment pour le Royaume-Uni d'un programme important ; chacun de ces deux pays avait donc son propre programme "SEABED" national.

Bien que pour la France, ce programme n'ait jamais été prioritaire et que le budget qui y a été consacré depuis le début ait été fort modeste, son rôle a été loin d'être négligeable. Elle a notamment effectué plusieurs campagnes océanographiques :

- en 1987, le Resolution de l'Institut français du pétrole (IFP) a effectué, pour le CEA, dans la zone des Bermudes, une couverture sismique profonde à haute résolution au moyen de la technique du Microflexichoc;

- en 1979, une nouvelle campagne sismique du Resolution a été réalisée pour le CEA dans la zone du Cap-Vert (sites CV1 et CV2) ;

- en 1980, le navire Jean Charcot du Centre national pour l'exploration des océans (CNEXO) (maintenant Institut français de recherches pour l'exploitation de la mer (IFREMER) est revenu sur les sites du CapVert, également pour le compte du CEA ; la campagne a consisté à effectuer des carottages de sédiments jusqu'à $15 \mathrm{~m}$, à procéder à des mesures sismiques au sondeur $3,5 \mathrm{kHz}$, à des levés bathymétriques au moyen du Seabeam (sondeur latéral) et à des mesures néphélémétriques; on a également effectué des relevés magnétiques et des mesures du flux thermique et mis en place des courantomètres pour la mesure des courants profonds ; on a, enfin, prélevé des échantillons de la faune profonde. 
En 1984, le CEA a proposé aux participants au programme SEABED de l'AEN d'organiser une grande campagne internationale dans l'Atlantique nord afin de recueillir le maximum d'informations sur deux points particulièrement importants du programme : la connaissance des caractéristiques des sédiments meubles des deux sites présélectionnés dans l'Atlantique nord, celui du Grand Météore Est (GME) et celui de la partie sud de la plaine abyssale de NARES (SNAP) et la capacité des torpilles à s'enfoncer sur plusieurs dizaines de mètres dans ces sédiments. La France disposait, en effet, pour cela, d'un carottier lourd à piston stationnaire mis au point par l'IFP, permettant de prélever des carottes non remaniées nécessaires pour effectuer des études géochimiques représentatives.

Le projet a suscité tout de suite un vif intérêt dans plusieurs pays ; l'opération a pu être montée en quelques mois et a pu être réalisée en juin-juillet 1985, grâce au navire des Terres australes et antarctiques françaises le "Marion-Dufresne", seul navire d'une dimension suffisante pour héberger les laboratoires et le personnel nécessaires pour la campagne. Un treuil nouveau a été installé à cet effet sur le navire, équipé d'un nouveau câble en kevlar de $\varnothing 29 \mathrm{~mm}$ et de 50 tonnes de charge de rupture effective, permettant d'économiser considérablement sur le poids. 70 scientifiques ont ainsi été embarqués durant 40 jours, appartenant à 6 pays différents : la France, les USA, le Royaume-Uni, le Canada, l'Italie et le Japon ainsi qu'au Centre de recherche commun d'Ispra de la CCE. 13 carottes ont été prélevées (5 sur GME dont une de $35 \mathrm{~m}$ et 8 sur SNAP dont 1 de $26 \mathrm{~m}$ ), sur lesquelles un très grand nombre d'investigations ont été menées tant à bord du Marion-Dufresne que, par la suite, dans des laboratoires à terre des différents pays. 17 torpilles instrumentées ont été lancées, dont 4 pour la France, ces dernières, équipées d'un système de transmission de données par fil, ont pénétré à GME à des profondeurs de $40 \mathrm{~m}$ dans les sédiments ( $30 \mathrm{~m}$ à SNAP). II a également été procédé à des prélèvements au carottier-boîte et à des levés sismiques.

Cette campagne internationale, la plus importante du programme SEABED, sans doute l'une des plus importantes jamais réalisées dans l'Atlantique nord quant au nombre de scientifiques embarqués, a apporté une abondance d'informations nouvelles. Les résultats scientifiques de la campagne jusqu'ici font l'objet d'un rapport de 1450 pages [2].

\section{RÉSULTATS DU PROGRAMME SEABED}

\section{RAPPORT DE FAISABILITÉ}

Les résultats de toutes les études effectuées dans le cadre du programme SEABED et le bilan des connaissances acquises à la fin du plan quinquennal 1983-1987 sont intégrés dans le rapport édité en 1988 par I'AEN "Faisabilité de l'évacuation des déchets de haute activité sous les fonds marins" [2]. Ce rapport comprend 8 volumes rédigés par des scientifiques impliqués dans le programme et appartenant aux différents 
pays concernés. II recouvre tous les domaines traités par les différents sous-groupes. Bien que l'option "forages" y soit examinée, le rapport concerne surtout l'option "torpilles". En effet, c'est surtout cette option qui a été développée tant en ce qui concerne l'investigation des sites potentiels que l'étude des techniques de mise en place. Le carottage à plusieurs centaines de mètres de profondeur des sédiments sous-marins et les expériences technologiques qu'aurait nécessité un examen élaboré de l'option "forages" étaient beaucoup trop onéreux pour les budgets disponibles dans les différents pays, à ce stade du programme. Le rapport se propose de répondre aux questions indiquées au début de cet article et qui constituent les objectifs du programme : l'existence de sites potentiellement favorables, la possibilité technique de mettre en place les déchets et la sûreté radiologique du dépôt. Nous ne nous étendrons pas ici sur les détails de ces questions qui remplissent 7 volumes pour nous limiter aux principaux résultats.

\subsection{Identification et sélection de sites favorables}

De nombreux sites potentiels ont été examinés tant dans le Pacifique que dans l'Atlantique nord. Pour cela, le groupe s'est donné, au départ, des critères de choix :

- épaisseur d'eau minimale d'environ $4000 \mathrm{~m}$,

- surface d'au moins $100 \mathrm{~km}^{2}$,

- topographie peu pentue,

- homogénéité horizontale et verticale des sédiments,

- érosion inférieure à $1 \mathrm{~m}$ en 250000 ans,

- pas d'activité volcanique ou sismique,

- pas de ressources naturelles,

- être situés hors d'atteinte des blocs erratiques transportés par les glaces dérivantes,

- épaisseur suffisante des sédiments : deux fois la profondeur d'enfouissement (on vise un enfouissement de $50 \mathrm{~m} \pm 20 \mathrm{~m}$ pour les torpilles et de $400 \mathrm{~m}$ dans le cas des forages),

- mouvement advectif des eaux interstitielles inférieur à $10^{-3} \mathrm{~m}$ par an,

- conditions géochimiques favorables et bonne capacité de sorption des sédiments,

- bioturbation des sédiments $<1 \%$,

- imperméabilité des sédiments.

Finalement, les critères principaux pris en compte sont ceux garantissant la possibilité de prédiction à long terme de la stabilité de la formation géologique et la capacité de barrière des sédiments. 
Treize sites ont été examinés dans l'Atlantique nord et cinq dans le Pacifique nord (fig. 1). Finalement, trois sites ont été retenus, un dans le Pacifique, le site E2, et deux dans l'Atlantique nord, SNAP (la partie sud de la plaine abyssale de NARES près des Antilles et GME (dans la plaine abyssale de Madère qui ont fait l'objet d'études plus approfondies, notamment, dans le cas de ces deux derniers, à la faveur de la campagne ESOPE (fig. 2) [1]. Ces sites sont ceux qui répondent le mieux aux critères définis précédemment, à la lumière des données qui ont pu être acquises jusqu'ici en ce qui concerne l'option "torpilles".

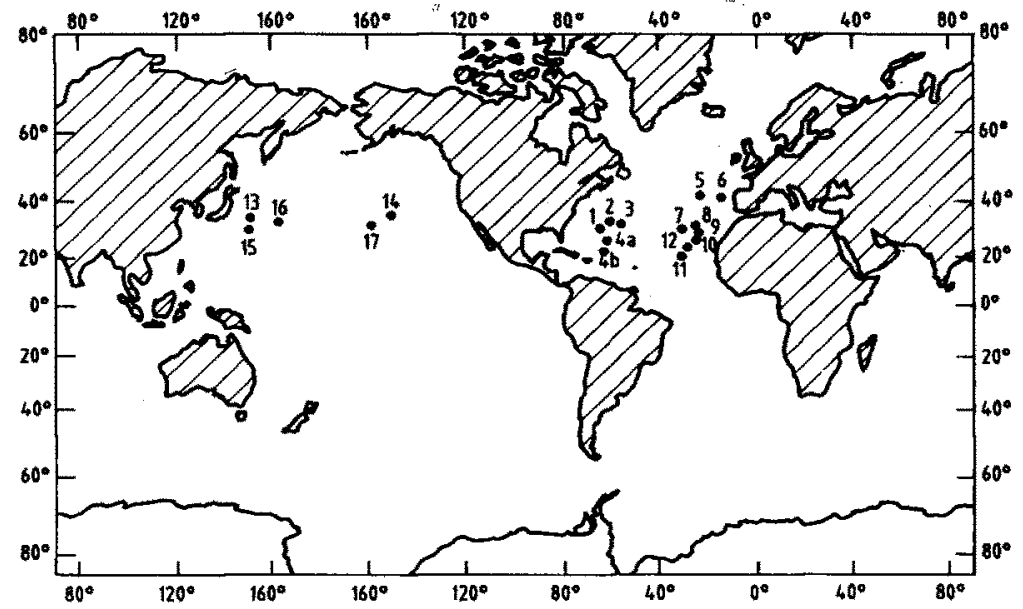

\begin{tabular}{|l}
\hline Atlantique Nord \\
8 (GME) Great Meteor East (Madeira Abyssal Plain) \\
5 (KTF) King's Trough Flank \\
$4 \mathrm{~b}$ (SNAP) Southern Nares Abyssal Plain \\
10 (CV1) Cape Verde Abyssal Plain (East) \\
11 (CV2) Cape Verde Rise \\
3 (SSAP) Southern Sohm Abyssal Plain \\
2 (NBR) Northern Bermuda Rise \\
7 (GMW) Great Meteor West \\
12 (CV3) Cape Verde Abyssal Plain (West) \\
4 (NNAP) Northern Nares Abyssal Plain \\
9 (Madcap) SW Madeira Abyssal Plain \\
1 (SBR) Southern Bermuda Rise \\
6 (IB1) Iberia \\
Pacifique Nord \\
13 (B1) PAC I (1100 km East of Japan) \\
14 (MPG I) PAC II (1900 km Northeast of Hawaii) \\
15 (CI) PAC II (1100 km Southeast of Japan) \\
16 (E2) PAC 1 (2000 km Southeast of Japan) \\
17 (MPG I) PAC II (1100 km North of Hawaii) \\
\hline
\end{tabular}

Fig. 1. 


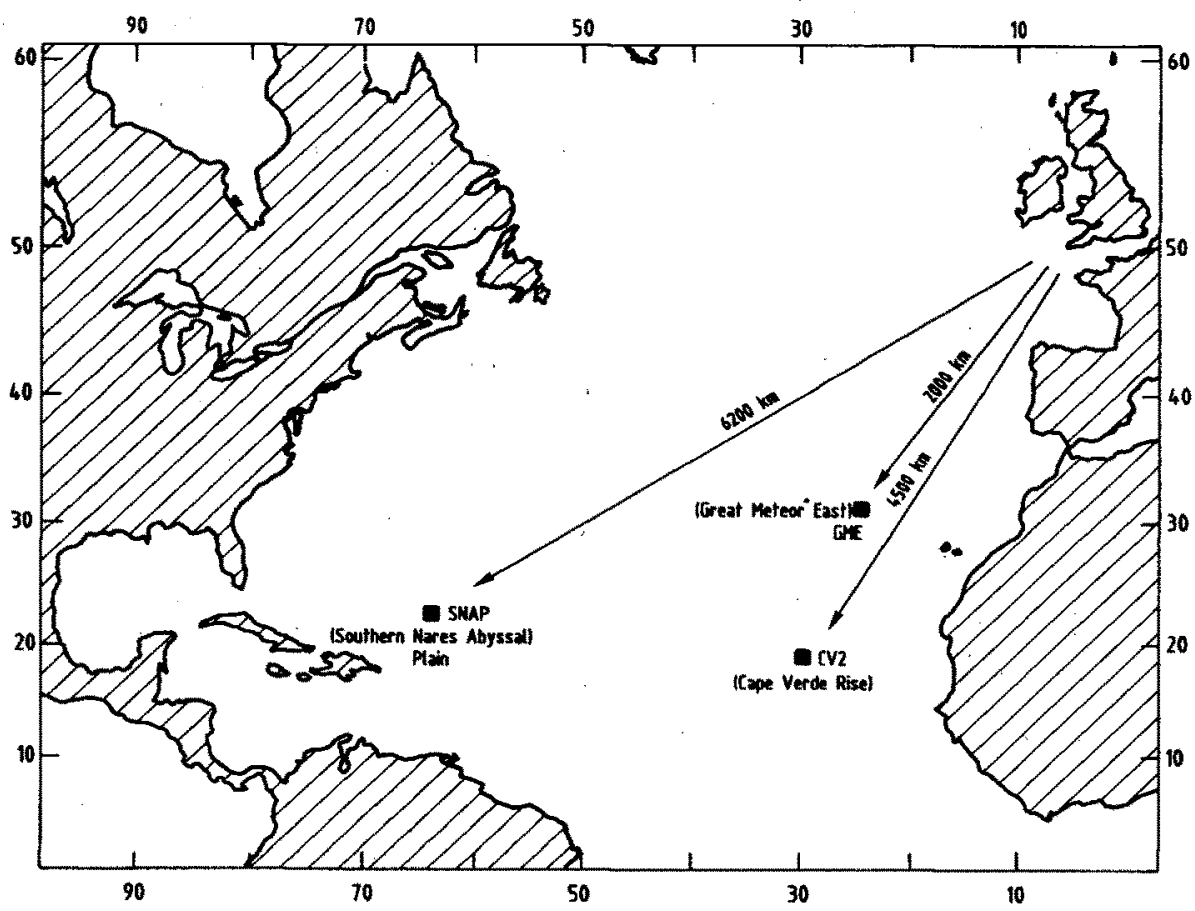

Fig. 2. - Zones en cours d'étude dans l'Atlantique nord.

En considérant plus particulièrement les sites de l'Atlantique auxquels se sont exclusivement intéressés la France et les autres pays européens, le site de GME est apparu comme un site potentiellement plus favorable que celui de NARES, car offrant des sédiments plus homogènes et de plus grande extension latérale. Les essais de torpilles ont montré également que, dans cette zone, les sédiments aux propriétés viscoplastiques favorables à la refermeture de la cheminée résultant de la pénétration pouvaient être aisément traversés jusqu'à $40 \mathrm{~m}$ par des torpilles à échelle réduite. De plus, ils n'apparaissent pas avoir été érodés de façon significative depuis 270000 ans et les études géochimiques ont montré que les mouvements advectifs des eaux interstitielles semblent inférieurs ou égaux au seuil de $10^{-3} \mathrm{~m} / \mathrm{an}$ correspondant à la vitesse de diffusion. On a, toutefois, mis en évidence des discontinuités ou failles qui paraissent résulter du tassement des sédiments et dont le rôle hydraulique devra être précisé par la suite.

A la première question, existe-t-il des sites favorables ? la réponse paraît donc aujourd'hui positive. 
3.2. Démonstration de la faisabilité technique (Possibilité de mise en place des conteneurs)

3.2.1. Forages (Fig. 3)

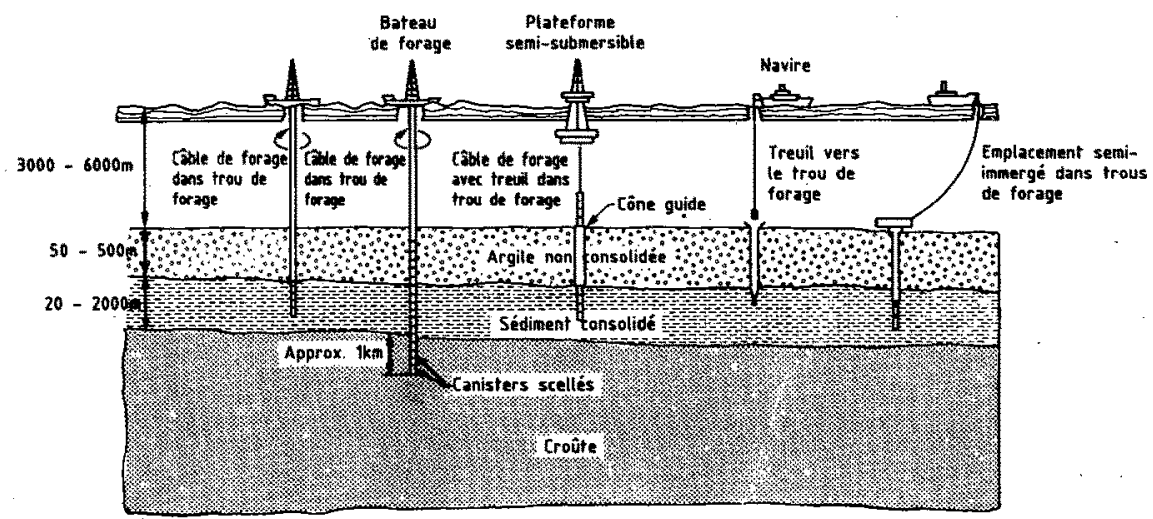

Fig. 3. - Concept de mise en place par forage.

Bien que cette option n'ait pas fait l'objet d'un examen très approfondi, le rapport conclut que, sur la base des techniques actuelles et par simple extrapolation, il peut être possible de mettre en place des conteneurs radioactifs dans des forages foncés jusqu'à plusieurs centaines de mètres dans des sédiments sous-marins consolidés et sous plus de $5000 \mathrm{~m}$ d'eau. De nombreuses techniques sont possibles pour la mise en place des conteneurs (fig. 3 et 4). Après réalisation du forage par un navire foreur, celle-cl pourrait se faire à partir d'une plate-forme positionnée au-dessus du trou. Une étude effectuée au Royaume-Uni propose de réaliser des forages profonds de $750 \mathrm{~m}$, qui seraient remplis de 300 conteneurs sur $500 \mathrm{~m}$ de haut. Une fois cette opération terminée, le navire foreur reviendrait pour retirer le cuvelage des 250 derniers mètres du trou et y placer un bouchon étanche.

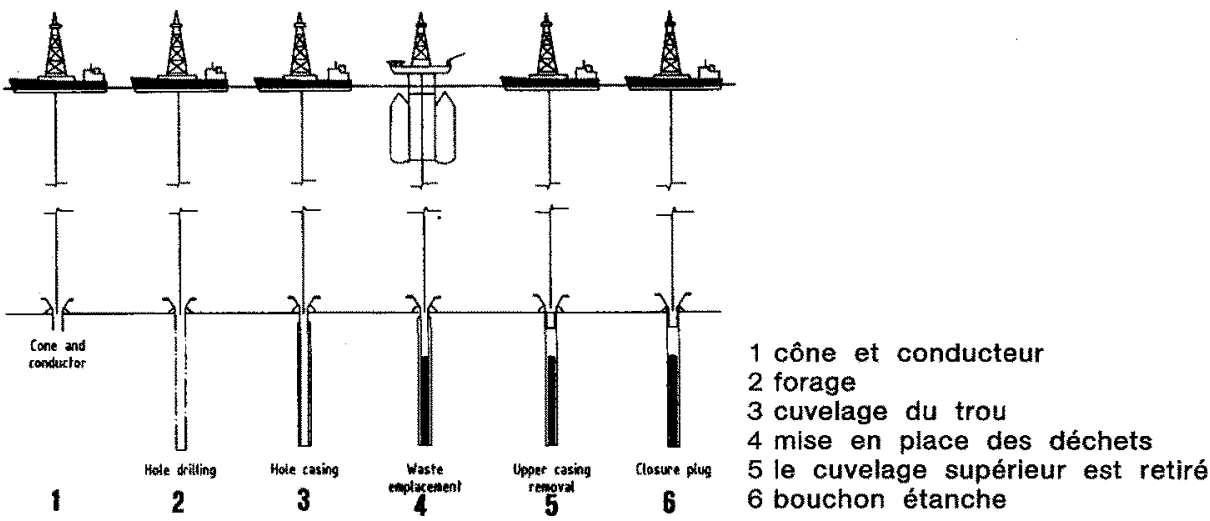

Fig. 4. - Séquence de mise en place des conteneurs. 


\subsubsection{Torpilles (pénétreurs)}

La mise en place des déchets au moyen de torpilles est plus simple et plus économique que la précédente. La solution la plus facile est de lâcher les torpilles depuis la surface et de les laisser tomber en chute libre; celles-ci s'enfoncent alors dans les sédiments grâce à leur énergie cinétique. D'autres solutions sont possibles (fig. 5). Les expériences réalisées in situ avec des torpilles instrumentées en modèle réduit (notamment lors de la campagne ESOPE) ont confirmé que celles-ci pouvaient atteindre des vitesses élevées et des profondeurs importantes $(53 \mathrm{~m} / \mathrm{s}$ et près de $40 \mathrm{~m}$ de profondeur à GME pour une torpille du CEA). On peut espérer que les torpilles "industrielles", beaucoup plus lourdes, atteindraient des vitesses de l'ordre de $60 \mathrm{~m} / \mathrm{s}$ et pourraient pénétrer à des profondeurs de 50 à $70 \mathrm{~m}$. Chaque lâcher de torpille serait espacé, de sorte que les différentes torpilles, une fois mise en place, n'interfèrent pas les unes avec les autres (notamment du point de vue thermique).

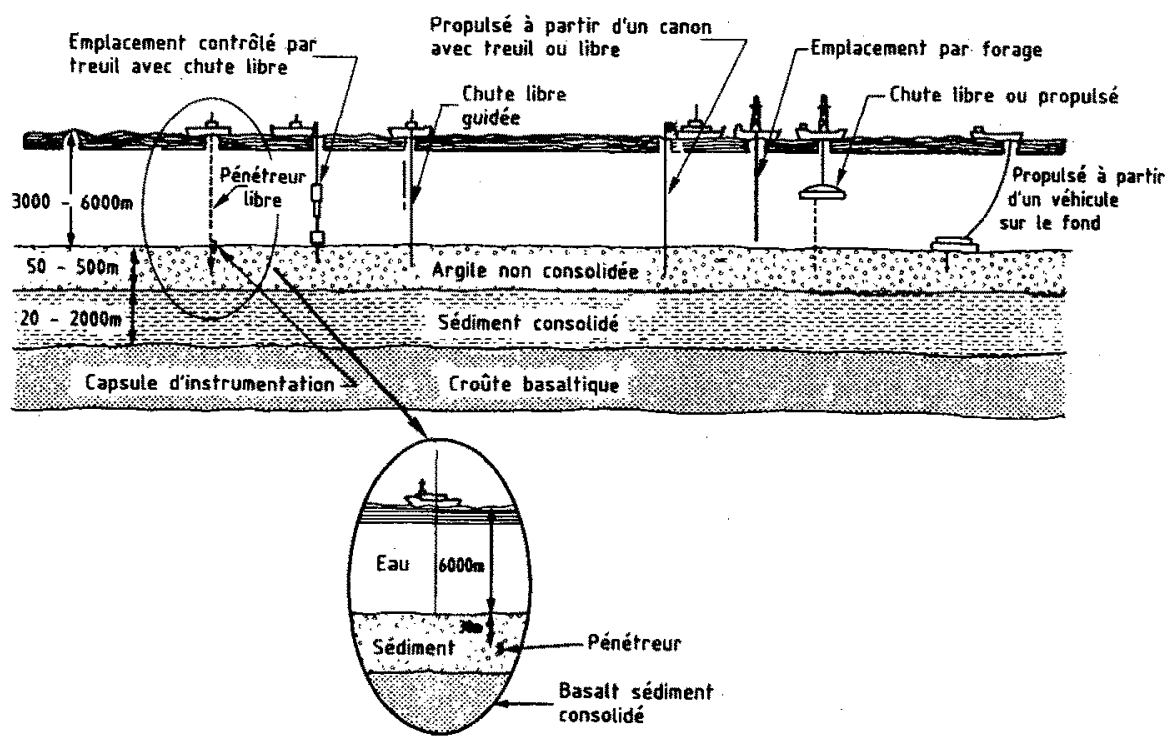

Fig. 5. - Concept de mise ne place par pénétreurs.

L'une des questions importantes soulevées par la mise en place des déchets par des torpilles est l'aptitude de la cheminée, creusée par l'engin dans les sédiments, à se refermer par la suite, reconstituant ainsi l'étanchéité du sédiment. Des considérations théoriques montrent que, par suite d'un phénomène dynamique (dépression à la queue de la torpille), le trou doit s'effondrer dès la pénétration. La plasticité des sédiments devrait faire le reste. Des expériences réalisées in situ au large des côtes françaises de la Méditerranée, bien qu'elles ne soient pas totalement concluantes, tendent à conforter cette hypothèse. 
Le rapport conclut donc positivement à la possibilité de mettre en place les déchets aux profondeurs requises a priori.

\subsection{Sûreté radiologique}

La sûreté radiologique est la troisième partie du triptyque de la faisabilité. Une opération de stockage de déchets radioactifs dans les formations géologiques sous-marines ne peut être envisagée que si l'on peut garantir qu'elle n'aura pas de conséquences néfastes sur le milieu vivant et sur l'homme.

Cela revient donc à s'assurer que les différentes barrières sont capables de jouer le rôle que l'on attend d'elles aussi longtemps que cela est nécessaire.

Les études visant à s'en assurer ont donc constitué une part importante du programme "SEABED". II s'agit d'identifier et d'examiner les différents processus susceptibles d'intervenir dans le confinement de la radioactivité ou, en cas de défaut de celui-ci, dans le transfert de celle-ci depuis le terme source jusqu'au milieu vivant.

Trois groupes de phénomènes doivent être pris en compte :

- ceux qui concernent les conteneurs et qui se déroulent à proximité des conteneurs (champ proche),

- ceux qui se rapportent à la migration des radionucléides à travers les sédiments,

- ceux qui concernent le devenir des radionucléides dans la colonne d'eau et dans le milieu vivant.

Ces différents processus ont fait l'objet d'études détaillées dans le cadre des différents sous-groupes concernés.

\subsubsection{Champ proche}

Le problème essentiel est évidemment la durée de vie du conteneur et de la matrice (verre) enrobant les déchets, qui conditionne le comportement du terme source. Il est souhaitable que celle-ci soit la plus longue possible et qu'elle couvre, en tous cas, au moins la période durant laquelle la charge thermique est susceptible d'engendrer des effets sur le milieu. Une durée minimale de 500 ans est souhaitée. Un autre groupe de problèmes concerne les modalités de transfert de chaleur dans les sédiments et les effets du champ thermique sur l'eau interstitielle (courants convectifs par exemple), sur la stabilité des colis de déchets et sur celle des sédiments eux-mêmes. Enfin, il convient aussi de savoir de quelle manière les effets du champ proche peuvent affecter les caractéristiques minéralogiques et géologiques du milieu. Les études ont porté sur les points suivants :

- la corrosion du conteneur : sur la base des tests réalisés, on peut conclure que les conteneurs devraient durer au moins le minimum de temps requis et, sous certaines conditions, pendant plusieurs millions d'années ; 
- la lixiviation de la matrice vitreuse : il semble que la vitesse de lixiviation ne soit pas linéaire : rapide au début, elle s'abaisserait sensiblement par la suite, en relation avec la concentration en silice; on espère que la matrice pourrait ainsi durer plusieurs milliers d'années avant d'avoir relâché tous les radionucléides qu'elle contient;

- les champs thermiques et radiatifs et leurs effets : les études effectuées ont montré que le transfert de chaleur à travers les sédiments s'effectue surtout par convection mais que les effets sur le milieu sont faibles tant en ce qui concerne le mouvement des conteneurs, le mouvement des sédiments que le mouvement des eaux interstitielles; les cellules convectives qui peuvent être ainsi générées resteraient d'extension très limitée ; de la même manière, les effets du rayonnement sur les sédiments et l'eau interstitielle paraissent peu importants.

\subsubsection{Migration des radionucléides à travers les sédiments}

La barrière sédimentaire étant considérée comme l'élément essentiel du confinement des radionucléides dans le concept SEABED, les phénomènes susceptibles d'intervenir dans la migration des éléments radioactifs ont été examinés avec attention. Les phénomènes quil peuvent entraîner le déplacement des radionucléides depuis le terme source jusqu'à l'interface eausédiments sont la diffusion et les mouvements d'eau interstitielle (advection) résultant soit de gradients thermiques naturels, soit de phénomènes de compaction.

Au stade actuel du déroulement du programme, il n'a pas été possible d'effectuer des mesures directes des mouvements de l'eau interstitielle. Ceux-ci n'ont pu être déduits qu'indirectement, à partir d'études géochimiques. On n'a pas pu mettre en évidence des mouvements advectifs d'une vitesse supérieure au seuil de $10^{-3} \mathrm{~m} / \mathrm{an}$. La vitesse de diffusion des radionucléides paraît très lente et considérablement réduite, pour la plupart d'entre eux, par adsorption sur la phase solide, quelles que soient les conditions minéralogiques et d'oxydo-réduction régnantes. Certains éléments tels que le carbone, l'iode, le sélénium et le technétium sont très peu retenus, mais il ne leur faudrait pas moins de 10000 ans pour traverser par diffusion les $50 \mathrm{~m}$ de couverture sédimentaire et atteindre les fonds de l'océan dans la masse duquel ils se dilueraient.

La couverture sédimentaire paraît donc bien jouer son rôle de barrière.

\subsubsection{Transport des radionucléldes dans la masse océanique}

Nous avons vu que l'un des avantages de la solution SEABED résidait dans les énormes possibilités de dilution offertes par la masse océanique dans le cas où des fuites de radionucléides pourraient se produire. II convenait, toutefois, d'étudier en détail le devenir de cette radioactivité dans la colonne d'eau. Celui-ci dépend du jeu de plusieurs facteurs : les modalités de circulation des eaux océaniques, le rôle des matières en suspension et le rôle des organismes vivants. 


\subsubsection{Océanographie physique}

Pour décrire la circulation des masses d'eau océanique et pouvoir calculer ainsi les champs de vitesse et les transferts de matière, on a développé plusieurs modèles permettant de prendre en compte les processus à petite échelle, à échelle moyenne et à grande échelle (modèle de circulation générale). L'ampleur et l'importance du travail à mener à bien n'ont pas permis de réaliser, dans le cadre du programme SEABED, compte tenu du temps imparti et des fonds attribués, un programme exhaustif aboutissant à une modélisation complète couvrant toutes les situations, appuyée sur des données suffisantes et validée par des expériences. II a, cependant, été possible de faire appel aux résultats d'autres programmes de recherches océanographiques qui ont ainsi permis d'étoffer les données disponibles.

La nécessité de disposer d'un modèle opérationnel pour effectuer les calculs d'évaluation de l'impact radiologique d'un stockage sur de très longues périodes de temps a conduit à élaborer un modèle simplifié ; ce modèle, le modèle Mark $A$, a été particulièrement développé par le Centre d'informatique géologique de l'Ecole nationale supérieure des mines de Paris, en collaboration avec des équipes internationales, dans le cadre d'un contrat avec l'IPSN/DPT. Ce modèle est un modèle à "boîtes" dans lequel l'océan Atlantique est découpé en 55 boîtes réparties tant horizontalement que verticalement. II inclut aussi la zone bioturbée et une boîte représentant les sédiments.

Le modèle Mark A concerne les processus suivants :

- la circulation advective générale dans l'océan, chaque boîte échange un flux d'une valeur donnée avec les boîtes adjacentes; cette valeur est tirée d'une liste de données compilées à cet effet ; lente :

- le flux dispersif entre les boîtes, afin de simuler la dispersion turbu-

- un modèle de sédimentation représentant le balayage dans la masse liquide des radionucléides par les particules en suspension et leur sédimentation sur le fond.

\subsubsection{Phéomènes biologiques}

Le concept SEABED minimise a priori l'importance de la biologie puisque la barrière sédimentaire est supposée confiner totalement la radioactivité contenue dans les déchets. Cependant, toute analyse d'impact reposant sur une hypothèse de fuite, les facteurs biologiques doivent être sérieusement pris en considération [1, vol. 5].

Tout d'abord, les organismes vivants peuvent, par l'intermédiaire de la chaîne alimentaire, constituer une route jusqu'à l'homme, consommateur de produits de la mer. Ensuite, les processus biologiques jouent un rôle significatif dans la distribution des radionucléides dans l'océan, tant dans l'espace que dans le temps. Cela peut résuiter soit d'actions directes telles que la perturbation des sédiments (bioturbation par exemple), soit de la production de particules en suspension qui fixent des radionucléides en solution 
et les précipitent sur le fond ou même peuvent en transformer l'état chimique et par conséquent le comportement ultérieur. Enfín, il convient, évidemment, de considérer l'impact potentiel sur la faune marine ellemême qui, comme tout le milieu vivant, doit être protégée.

II convient donc d'étudier de quelle façon la radioactivité peut être transportée et concentrée par les organismes marins. De nombreuses études ont été réalisées dans ce domaine dans le monde tant dans le cadre du programme SEABED que dans le cadre de programmes différents, notamment le programme IMMERSION, piloté également par l'AEN, pour lequel les aspects biologiques sont particulièrement importants. Ces résultats ont pu être utilisés dans le programme SEABED.

Des études ont porté sur l'identification et la répartition des faunes abyssales, dans lesquelles les espèces carnassières et nécrophages, telles que les amphipodes, jouent un rôle très important, d'autant que ce sont des animaux très mobiles. Les écosystèmes profonds ont été étudiés ; des études ont été effectuées sur l'absorption d'oxygène et sur les échanges nutritifs. On a pu ainsi mettre en évidence des variations saisonnières. Le plancton proche du fond peut contribuer aux transferts biologiques par suite de son métabolisme cumulatif élevé. Des études ont été effectuées sur les facteurs de transfert de l'amphipode Eurythènes gryllus au moyen de pièges posés sur le fond et d'appâts marqués ; on a pu, de la sorte, mettre en évidence le taux d'assimilation des matières organiques de cette créature ; elle paraît avoir un transit digestif lent. Des études ont également porté sur les micro-organismes présents dans les sédiments. Si les bactéries paraissent surtout prospérer dans les premières dizaines de centimètres, les carottages de la campagne ESOPE ont montré que l'on pouvait en rencontrer beaucoup plus profondément. L'effet de la chaleur sur ces bactéries reste à examiner ; on pense, cependant, actuellement, que l'élévation de température résultant de la charge thermique des conteneurs n'aura pas de conséquences appréciables.

II est également important d'étudier les échanges sédiments-eau. A travers la masse d'eau océanique, les échanges peuvent être considérés comme actifs ou passifs : le transport passif résulte de la sédimentation des particules organiques tandis que le mode actif résulte du transport par les organismes vivants.

Si la faune mésopélagique peut entraîner des migrations verticales, il y a peu d'évidence qu'il y ait des échanges importants avec les eaux profondes incluant l'interface eau-sédiments. D'une façon générale, l'évaluation de la biomasse est un des aspects essentiels des études de biologie marine tant en ce qui concerne la microfaune, la mélofaune que la mégafaune. La biomasse benthique est très faible.

Les principales voies biologiques de transport des radionucléides jusqu'à l'homme sont :

- les phénomènes de bioturbation des sédiments ; 
- la migration d'animaux depuis le fond jusqu'aux eaux peu profondes, cas, par exemple, des holothuries pélagiques ; il s'agit, toutefois, de quantités si faibles qu'elles ne peuvent pas contribuer de façon significative à un flux vertical de matière ;

- le transport par l'intermédiaire de prédateurs ; les cachalots, par exemple, sont susceptibles d'aller chercher des proies jusqu'à des profondeurs de $3000 \mathrm{~m}$, constituées principalement de calmars ; par ailleurs, les céphalopodes peuvent aussi être utilisés directement comme nourriture par l'homme ; fond.

- la remontée de particules biologiques, d'œufs ou de larves depuis le

Par ailleurs, la faune bentho-pélagique qui vit près du fond peut, dans certaines régions, interagir avec la faune pélagique assurant, de la sorte, une certaine continuité avec la faune du glacis et du talus continental. $\mathrm{Ce}$ phénomène paraît, toutefois, limité.

Aucune de ces voies de transfert n'apparaît actuellement comme vraiment importante, à la lumière des modélisations effectuées. II semble, dans l'état actuel de nos connaissances, que le risque principal de contamination pourrait résulter de la consommation de produits de la mer provenant des eaux côtières contaminées par suite de processus advectifs ou diffusifs affectant les eaux océaniques plutôt que résultant d'un transfert biologique. La consommation directe d'espèces de la mégafaune profonde éventuellement contaminée par une fuite du dépôt paraît assez peu vraisemblable, ne serait-ce que par suite de la très faible densité de ces animaux.

Des études ont également été effectuées sur la contamination et la sensibilité aux rayonnements des organismes marins. On a ainsi examiné les voies par lesquelles elle pouvait cheminer et les tissus dans lesquels elle pouvait se concentrer, la sensibilité des organismes aux différentes formes chimiques radioactives et les facteurs de concentration des radionucléides dans les différentes espèces. Les espèces profondes ont été étudiées au moyen de modules de colonisation posés sur le fond. On a également réalisé des études sur des espèces peu profondes, d'expérimentation plus aisée ; celles-ci n'en sont pas moins pertinentes du fait que ce sont surtout ces espèces qui sont consommées par l'homme.

En ce qui concerne les radionucléides naturels, on observe une concentration en ${ }^{210}$ Po qui passe du phytoplancton et du zooplancton aux poissons qui s'en alimentent (concentration surtout dans le foie, mais aussi dans les gonades). En ce qui concerne les radionucléides artificiels, ${ }^{137} \mathrm{Cs}$ trouvé dans l'espèce Coryphénoldes armatus paraît en relation avec les retombées atmosphériques.

Enfin, en ce qui concerne les effets des rayonnements sur la faune profonde, on a étudié l'effet des rayonnements $\alpha, \beta, y$. L'irradiation externe (résultant de ${ }^{40} \mathrm{~K}$ et de ${ }^{87} \mathrm{Rb}$ dans l'eau) paraît équivalente pour la faune de fond et la faune de surface. Au contact du fond, elle dépend surtout de la teneur des sédiments en ${ }^{238} \mathrm{U}$ et ${ }^{232} \mathrm{Th}$ et leurs produits de filiation. Les effets devraient être insignifiants. 
En conclusion, il apparaît que l'un des rôles des organismes marins est de produire des particules de matières susceptibles de fixer la radioactivité contenue dans l'eau et de la reprécipiter sur le fond. Sur la base des connaissances jusqu'ici acquises, aucun facteur biologique n'est donc apparu qui puisse condamner l'option SEABED.

\subsubsection{Analyse des conséquences radlologiques}

La connaissance des différents processus qui viennent d'être passés rapidement en revue est nécessaire pour permettre de définir les scénarios et d'élaborer les modèles qui permettront de déterminer les conséquences radiologiques du dépôt sur le milieu vivant et sur l'homme, donc de s'assurer qu'une opération d'évacuation des déchets radioactifs sous les fonds marins est acceptable du point de vue de la sûreté et répondre ainsi à la dernière des trois questions fondamentales.

L'étude de sûreté a porté sur les sites de NARES (SNAP) et du Grand Météore (GME) dont les résultats ont été, d'ailleurs, tout à fait comparables.

Trois situations ont été envisagées, en ce qui concerne les scénarios :

1 - un scénario normal de référence dans lequel les différents processus sont conformes aux prévisions (profondeur d'enfouissement, comportement des barrières etc.) ;

2 - des scénarios anormaux dans lesquels certains composants du système se comportent anormalement (par exemple : érosion réduisant l'épaisseur des sédiments de couverture, présence de failles, mouvements advectifs des eaux interstitielles vers la surface, enfouissement des conteneurs à des profondeurs moindres etc.) ;

3 - accidents de transport.

Les modèles utilisés pour les calculs sont ceux développés par les différents groupes techniques sur la base des connaissances acquises des différents phénomènes et processus intervenant dans le terme source et les transferts de radioactivité. Ils concernent donc :

- la corrosion des conteneurs,

- la lixiviation des verres,

- le transport des radionucléides à travers la barrière sédimentaire,

- la dispersion des radionucléides dans la masse océanique,

- le "balayage" de certains radionucléides et leur sédimentation, par adsorption sur des particules de sédiments ou des matières organiques en suspension,

- les différentes voies de transfert physique ou biologique jusqu'à I'homme (produits de la mer, sel, aérosols, irradiation, etc.). 
Les calculs ont été effectués sur la base des hypothèses suivantes :

- retraitement de 100000 tonnes de métal lourd représentant les déchets produits par 3333 réacteurs-an de $900 \mathrm{MWe}$,

- entreposage pendant 50 ans,

- stockage de 14667 torpilles contenant chacune 5 conteneurs, à $5 \mathrm{~m}$ de profondeur dans les sédiments et disposées à $180 \mathrm{~m}$ les unes des autres, la zone de stockage couvre ainsi $484 \mathrm{~km}^{2}$.

Dans le cas normal de référence, la dose maximale au groupe critique d'individus est de l'ordre de $3.10^{-9}$ Sv/an. Elle se produit 150000 ans après la mise en place des déchets (fig. 6 et 7). Les radionucléides qui contribuent le plus à cette dose sont ${ }^{99} \mathrm{Tc},{ }^{79} \mathrm{Se},{ }^{126} \mathrm{Sn},{ }^{129} \mathrm{I},{ }^{135} \mathrm{Cs}$. Les voies critiques sont principalement la consommation de mollusques, de crustacés, d'algues et de polssons. Ces doses sont très inférieures à la dose limite recommandée par la CIPR $\left(10^{-3}\right.$ Sv/an) et au rayonnement naturel $\left(10^{-3} \mathrm{~Sv} / \mathrm{an}\right)$. Les doses collectives intégrées sont respectivement $2.10^{3}$ homme-sievert à 100000 ans : (contribution : ${ }^{79} \mathrm{Se}$ pour $80 \%$, ${ }^{99} \mathrm{Tc}$ pour $19 \%$ ) et $3.10^{4}$ homme-sievert à 10 millions d'années (contribution : ${ }^{135} \mathrm{Cs}$ pour $56 \%,{ }^{79} \mathrm{Se}$ pour $24 \%$, ${ }^{94} \mathrm{Tc}$ pour $18 \%$ ).

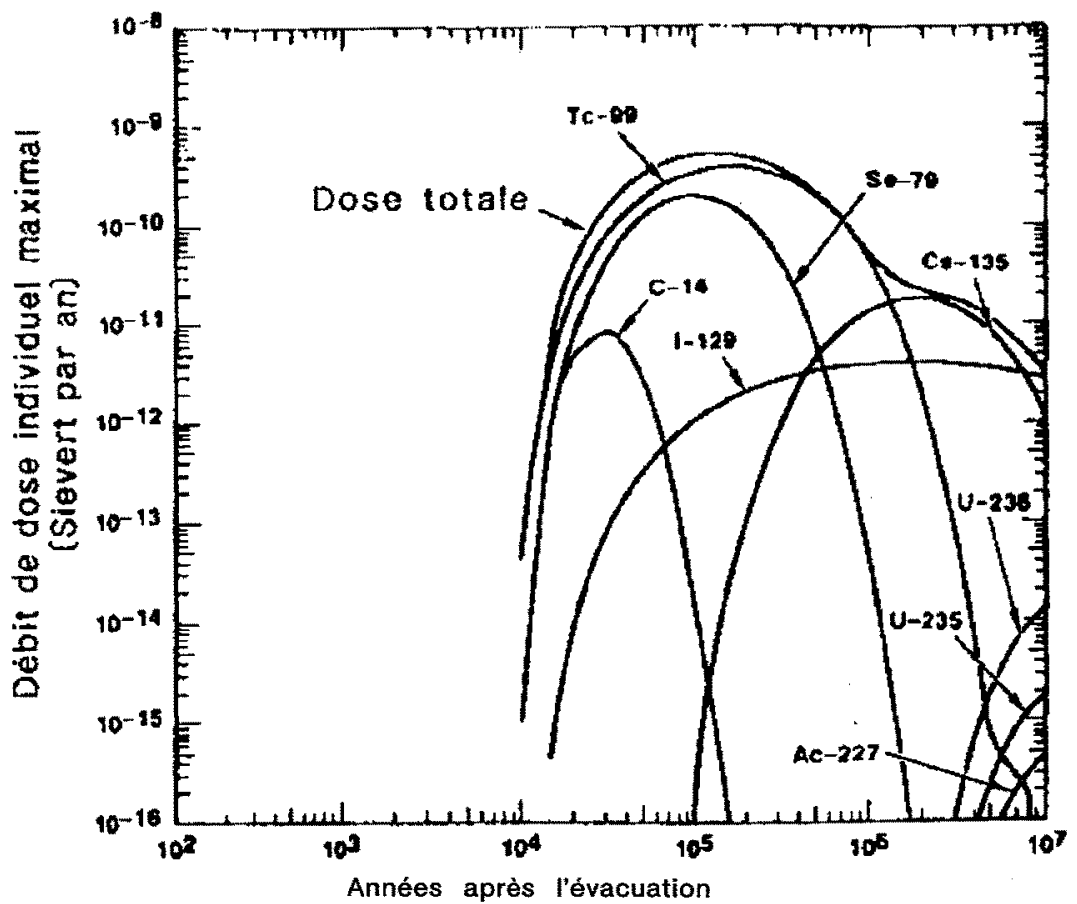

Fig. 6. - Débit de dose individuel maximal par an pour le cas de référence, à partir d'une analyse déterministe appliquée au modèle à compartiments Mark-A. Ce cas repose sur des valeurs réalistes des paramètres. Les courbes situées sous la courbe représentant la dose totale correspondent aux contributions relatives des divers radionucléides. 


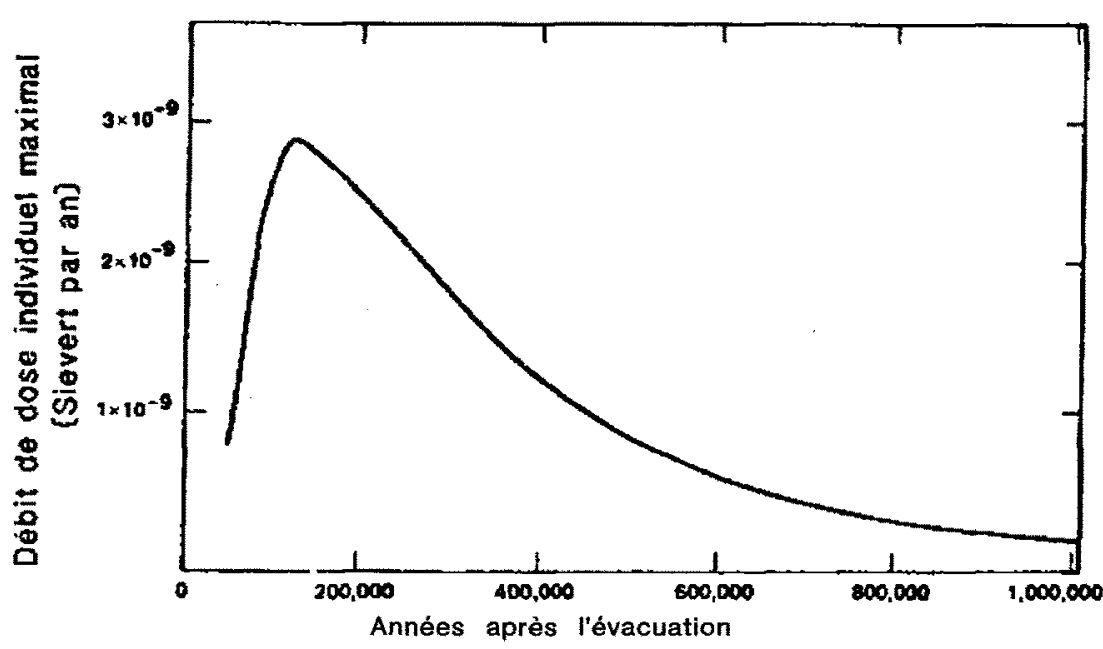

Fig. 7. - Débit de dose individuel maximal par an pour le cas de référence tel qu'il résulte d'une analyse probabiliste.

En ce qui concerne les scénarios anormaux, les analyses de sensibilité paramétrique montrent que, seuls, 3 scénarios contribuent à accroître les doses :

- si les torpilles sont enfouies à moins de 10 mètres de profondeur,

- dans le cas de phénomènes d'advection appréciable entraînant une circulation des eaux interstitielles vers la surface,

- dans le cas où la rétention serait nulle pour tous les radionucléides.

En ce qui concerne les scénarios accidentels, les doses les plus sévères sont obtenues dans le cas de naufrage du navire transportant les conteneurs. Les bateaux effectuant ce genre de transport devront donc être conçus pour être à l'abri de ce genre de risque.

Le concept SEABED paraît donc bien présenter, à la lumière des données actuelles, une très large marge de sûreté ; mais il est évident que des études doivent être poursuivies dans certains domaines (notamment la circulation des eaux interstitielles dans les sédiments) pour lever les incertitudes qui peuvent encore demeurer et valider les hypothèses, les données et les modèles utilisés.

\section{CONCLUSIONS}

Aux trois questions que l'on se posait au début du programme :

- existe-t-il des sites favorables ?

- peut-on techniquement mettre les déchets en place dans les sédiments ?

- l'opération est-elle sûre ? 
onze ans de recherches dans un cadre international ont permis de répondre par l'affirmative, à la lumière des informations disponibles. L'option SEABED apparaît donc, actuellement, comme la solution alternative la plus intéressante aux stockages en formation géologique continentale. Par ailleurs, les premières évaluations de coût montrent qu'elle serait, sans doute, également très compétitive sur le plan économique. Enfin, il faut souligner que le programme SEABED aura été particulièrement exemplaire dans le domaine de la coopération internationale.

\section{RÉFÉRENCES}

[1] Geoscience investigation of two North-Atlantic abyssal plains, the esope international expedition. Ispra : Joint research centre, 1989, 2 vol. (EUR-12330 et EUR-12331).

[2] OCDE. AGENCE POUR L'ÉNERGIE NUCLÉAIRE (AEN). - Feasibility of disposal of high-level radioactive waste into the seabed. Paris: OCDE, 1988, 8 vol. 